\title{
Infraestrutura de acesso em redes sem fio obstruídas: da Intratabilidade à Conectividade
}

\author{
Manassés Ferreira Neto ${ }^{1}$, Olga Nikolaevna Goussevskaia ${ }^{1}$, \\ Vinicius Fernandes dos Santos $^{1}$ \\ ${ }^{1}$ Departamento de Ciência da Computação \\ Universidade Federal de Minas Gerais (UFMG) \\ \{manasses, olga, viniciussantos\}@dcc.ufmg.br
}

\begin{abstract}
Resumo. Neste trabalho é considerada uma rede sem fio ad hoc realizada em uma grade regular quadrada, na qual a comunicação entre os dispositivos é influenciada por obstáculos regularmente espaçados. $O$ raio crítico de transmissão para obter conectividade nesse tipo de rede cresce com o tamanho da grade, o que pode prejudicar a viabilidade em larga escala de tecnologias sem fio de baixa potência. Avalia-se portanto como introduzir eficientemente uma infraestrutura conectada de pontos de acesso em cenários subcríticos, nos quais o raio de transmissão é insuficiente para estabelecer a conectividade. Formula-se o problema de posicionar o menor número de pontos de acesso, de tal modo que todo componente conectado seja coberto por pelo menos um ponto de acesso, e denomina-se esse problema de Obstructed Wireless Network Backbone Cover Problem (OWN-BC). Prova-se que OWN-BC é NP-Completo e propõe-se um algoritmo 2-aproximativo para obter soluções com garantia de qualidade. Realiza-se simulações para ilustrar o desempenho do algoritmo em diferentes cenários. Além disso, é feita uma caracterização de cenários para os quais o algoritmo proposto obtêm soluções ótimas em tempo polinomial com alta probabilidade.
\end{abstract}

\section{Connectivity with backbone structures in Obstructed Wireless Networks}

In this work we explore the limits of connectivity of wireless networks performed in environments with obstacles - the so-called Obstructed Wireless Networks (OWN). Obstacles are present in a variety of networking application scenarios, such as vehicular networks operating in urban street grids, home networks, or other networks deployed indoors, tunnels, or underground mines. Let consider, for instance, the formation of a network only between the cars located on the streets of a city with many buildings, like New York City. The described vehicular network does not rely on a pre-existing infrastructure, being called ad hoc.

We start this work by analyzing the scalability of ad hoc wireless networks obstructed by regularly spaced obstacles, using the model proposed in [Almiron et al. 2013]. In this model, the street blocks of the city are represented as squares on a two-dimensional grid and the devices, as points on uni-dimensional streets.A device has the communication range dependent on the wireless technology applied. A pair of devices are connected if they meet specific communication rules, roughly, a function of distance and the absence of obstacles between them. A set of connected devices forms the so-called connected component, or just, component. 
The main contribution of this work is the study of scenarios where either the number of devices or its communication range are not large enough to ensure overall connectivity and the network is partitioned into several components. We assume that a global communication infrastructure exists, e.g. the Internet, which can be accessed through one or more access points by any device, as long as there is a path of communication links from that device to at least one access point to the infrastructure. This infrastructure, also referred in this work as backbone, provides connectivity between the ad hoc components. We enumerate all the candidate positions for access points. And finally, we obtain the minimum set of access points, selected from the candidates, to establish connectivity.

We define the Obstructed Wireless Network Backbone Cover (OWN-BC) problem. We prove that, given an arbitrary device distribution on the grid, OWN-BC is NPcomplete and propose a 2-approximation algorithm to compute close-to-optimum solutions in polynomial time. We simulated diverse OWN scenarios and observed that the obtained solutions were remarkably close to the optimum, which motivated us to analyze the complexity of typical problem instances. Because of this, we turn our attention to the randomly-generated device distributions. By analyzing the structure of the problem in random scenarios, we showed that it can be solved optimally in polynomial time, as long as certain criteria are satisfied at the intersections of the grid structure. In particular, we present an analytical lower bound on the probability of the approximation algorithm to compute optimum solutions in polynomial time. We validate this analytical result by simulating the empirical probability of finding optimum solutions in polynomial time in sub-critical network configurations. The simulations corroborated the good quality of the analytical result.

We believe that, from a practical point of view, these are potentially valuable results, since they allow (a communication engineer) to ensure connectivity in OWN with arbitrary device deployment, with or without a backbone, for any network size, communication technology, or geometry of a particular obstacle grid. Moreover, it is possible to identify scenarios where optimal backbone solutions can be quickly computed.

\section{Related work}

Relatively few attempts have been made to analyze OWN, many of which are quite complex and not easily generalizable.The Random OWN model was proposed in [Almiron et al. 2013], with the objective to characterize the Critical Transmission Range (CTR) for connectivity.

\section{Model}

In order to study obstructed wireless networks, we need a model that captures some essential characteristics of obstructed environments encountered in practice and is simple enough to provide analytical tools for network properties, such as connectivity. The OWN model is defined as follows.

Definition 1. Obstructed Wireless Network $(O W N)$ : An instance of an $O W N$ is defined by the following parameters: grid size $g$, street width $\epsilon$, communication range $r$, and device set $D$. As illustrated by Figure la, device deployment is done over the $g \times g$ grid. Each street is comprised of $g-1$ blocks, to which we refer as segments and $g$ crossroads, to which we refer as intersections. The normalized length of a segment is 


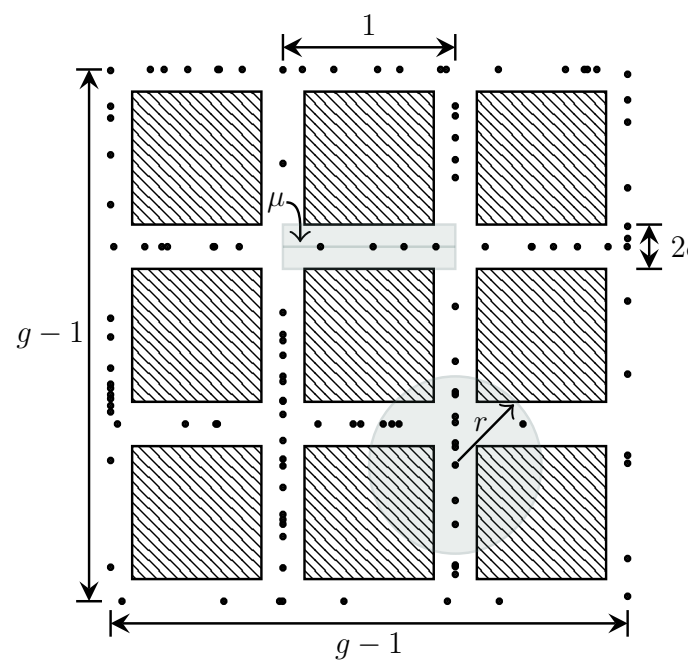

(a) Grid layout.

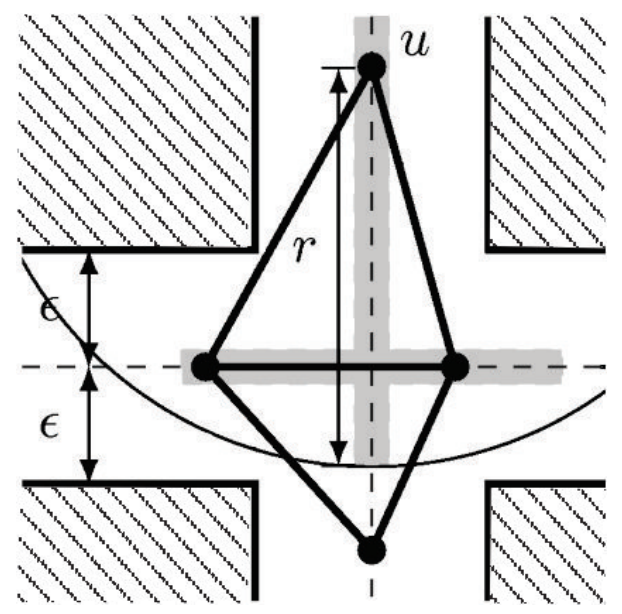

(b) LoS: The gray region is the coverage of device $u$

Figure 1: OWN model: deployment and connectivity.

set to one, and the street width is set to $0<2 \epsilon<1$. The communication devices in $D$ are positioned at arbitrary coordinates, but always on uni-dimensional and centralized lines along street segments. The communication links are established between any pair of devices $(u, v) \in D$, that meet two criteria: (1) Euclidean distance: $d(u, v) \leq r$; and (2) Visibility: there is no obstacle between $u$ and $v$, i.e., there is Line-of-Sight (LoS) (see Figure $1 b$ ).

Below, we define a probabilistic version of the OWN model, which uses a uniformly distributed device deployment and was first introduced in [Almiron et al. 2013].

Definition 2. Random OWN: In addition to the parameters defined in Definition 1, the Random OWN uses the parameter node density $\mu$. The deployment of devices in set $D$ is performed in the following manner: in each street segment of the grid, $\mu$ communication devices are deployed uniformly at random (on uni-dimensional and centralized lines). Therefore, $|D|=2 g(g-1) \cdot \mu$.

Definition 3. CTR for Connectivity: Suppose $n$ devices are distributed in a deployment region. The Critical Transmission Range (CTR) for connectivity can be defined as the minimum transmission range, denoted by $r_{c}$, which induces a communication graph with a unique connected component, including all $n$ devices.

In [Almiron et al. 2013] discrete percolation theory elements were applied to study global network properties, such as the formation of a giant connected component and the CTR for connectivity. Therefore, the CTR is a threshold, beyond which connectivity is warranted with high probability (w.h.p.) (super-critical scenarios), and under which the network becomes fragmented, or partitioned into several connected components (sub-critical scenarios). It can be obtained as a function of the models parameters as stated in Theorem 1.

Theorem 1. ([Almiron et al. 2013]) Given a Random OWN, the CTR for connectivity w.h.p., denoted by $r_{c}$, is

$$
r_{c}=\frac{\ln \left(g^{a+1 / 2}\right)+\ln (\mu-1)}{\mu}
$$


for $a>0$, whenever $\epsilon \geq \epsilon_{c}$.

The value $\epsilon_{c}$ is the so-called critical width, i.e., the minimal value of $\epsilon$ that guarantees the probability of connectivity at street intersections. Any positive value of parameter $a$ induces connectivity when $g \rightarrow \infty$, and higher values can be used for faster convergence according to the specific value of $g$.

\section{Main contributions}

We point out briefly our main contributions. Due the lack of space, we refer the reader to the full article for detailed explanation [Neto et al. 2017].

\subsection{Scalability of connectivity in ad hoc Obstructed Wireless Networks}

We presented a model that can be easily adjusted for real communication tecnologies proportions. For such, expression (1) computes the CTR for connectivity, for a given grid size $g$ and device density $\mu$ in a ad hoc Random OWN. Alternatively, we can think about the transmission range as a constant $\tilde{r}$ and determine, afterward, the scalability of the network in terms of size.

Note that when $g \rightarrow \infty$, an infinite amount of connected components emerges, independently of the value of $\tilde{r}$. As a consequence, for a fixed value of $\tilde{r}$, we have a restriction on values for $g$ and $\mu$. More specifically, big values of $g$ require higher values of $\mu$. This relationship is given by manipulating the expression (1) as follows (whenever we use the convergence factor $a=1$ ):

$$
g=\left(\frac{e^{\mu \tilde{r}}}{\mu-1}\right)^{\frac{2}{3}}
$$

In order to demonstrate the practical implications of Expression (2), let us take as an example typical values of transmission range for the IEEE 802.15.4 and 802.11 legacy standards in a "Standard City" with street blocks of size $100 \mathrm{~m}$. For instance, a network with IEEE 802.15 .4 devices configured to transmit up to $30 \mathrm{~m}$ at $250 \mathrm{kbps}$ requires a dense deployment, about 30 devices per segment, to be able to scale to a grid of $42 \times 42$, while maintaining connectivity. On the other hand, if communication technology can be updated such that the transmission range is $40 \mathrm{~m}$, with the same device density, connectivity is achieved w.h.p. in much larger urban areas, with grid sizes up to 315 .

It can be inferred that small variations in the configuration of transmission range have a high impact on the scalability of connectivity in ad hoc OWN. To sum up, if some parameters such as the radio transmission range are restricted connectivity doesn't scales well with the size of the grid, the necessary device density explodes to meet the connectivity requirement. This behavior points to a necessity of other network topologies, such as connected backbone structures, in order to obtain connectivity in OWN.

\subsection{Obstructed Wireless Network Backbone Cover problem}

We are interested in the following problem, formally defined below.

Definition 4. OWN Backbone Cover Problem (OWN-BC): Consider an OWN, partitioned into a set of components $C$. Consider a set $\mathcal{B}$ of candidate locations for access points to 


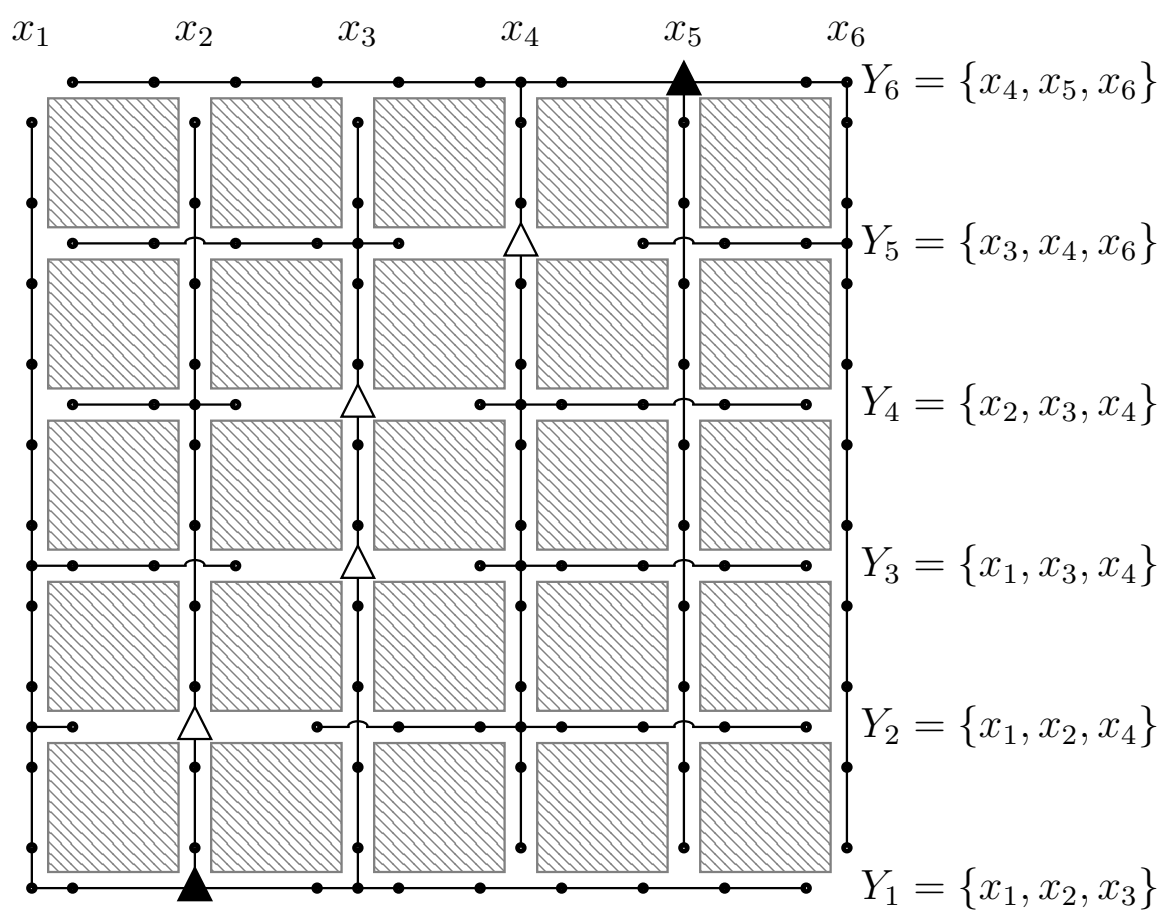

Figure 2: $\mathrm{X} 3 \mathrm{C}$ to OWN-BC reduction example: $X=\left\{x_{1}, x_{2}, x_{3}, x_{4}, x_{5}, x_{6}\right\}, Y=\left\{Y_{1}=\right.$ $\left\{x_{1}, x_{2}, x_{3}\right\}, Y_{2}=\left\{x_{1}, x_{2}, x_{4}\right\}, Y_{3}=\left\{x_{1}, x_{3}, x_{4}\right\}, Y_{4}=\left\{x_{2}, x_{3}, x_{4}\right\}, Y_{5}=\left\{x_{3}, x_{4}, x_{6}\right\}$, $\left.Y_{6}=\left\{x_{4}, x_{5}, x_{6}\right\}\right\}$.

the backbone, and denote by $\mathcal{B}\left(C_{j}\right) \subseteq \mathcal{B}, C_{j} \in C$, the subset of candidate locations that cover each component of the OWN. The objective of the OWN-BC is to connect every component to the backbone by activating at least one access point in $\mathcal{B}_{i} \in \mathcal{B}\left(C_{j}\right) \subseteq$ $\mathcal{B}, \forall C_{j} \in C$, while minimizing the total number of active access points:

$$
\begin{aligned}
& \min \sum_{\mathcal{B}_{i} \in \mathcal{B}} b_{i} \\
& \text { s.t. } \quad \sum_{\mathcal{B}_{i} \in \mathcal{B}\left(C_{j}\right)} b_{i} \geq 1, \quad \forall C_{j} \in C \\
& b_{i} \in\{0,1\}, \quad \forall \mathcal{B}_{i} \in \mathcal{B} .
\end{aligned}
$$

We prove that OWN-BC is NP-hard by reduction from the Exact Cover by 3-Sets (X3C) Problem [Garey and Johnson 2002], which is a classical NP-complete problem, formulated as follows.

Definition 5. Exact Cover by 3-Sets Problem (X3C): Given a set $X=\left\{x_{1}, x_{2}, \ldots, x_{3 q}\right\}$ and a collection $Y=\left\{Y_{1}, Y_{2}, \ldots, Y_{|Y|}\right\}$, such that $Y_{i}$ is a 3-element subset of $X, X 3 C$ consists in deciding whether there is an exact cover of $X$ by sets of $Y$ (i.e., if it is possible to select mutually disjoint sets from $Y$ such that their union is exactly $X$ ).

We outline in Figure 2 how an instance of $\mathrm{X} 3 \mathrm{C}$ is reduced, in polynomial time, to an instance of OWN-BC, the decision version of OWN-BC, such that the existence of solution on the latter implies the existence of a solution on the former, and conversely. We consider a one-to-one relation between each row (horizontal street) of the OWN-BC instance and a 3-set $Y_{j} \in Y$ in the $\mathrm{X} 3 \mathrm{C}$ instance. We indicate the reader to [Neto et al. 2017] for the full proof. 
Since OWN-BC is NP-complete, unless $P=N P$, there is no polynomial-time algorithm to solve arbitrary instances. Therefore, we discuss approximation algorithms for the problem.

\subsection{Approximation algorithm}

The OWN-BC problem can be modeled as the Set Cover problem, where network components correspond to the elements and access points to the sets. Given that an access point to the backbone can assist at most 4 disconnected network components, it can be viewed as a special case of the $\mathrm{k}$-Set Cover (k-SC) problem, where $k=4$. The $k$-Set Cover can be defined as follows.

Definition 6. $\boldsymbol{k}$-Set Cover Problem (k-SC): Given a set of elements $X=$ $\left\{x_{1}, x_{2}, \ldots, x_{n}\right\}$, and a set $C=\left\{C_{1}, C_{2}, \ldots, C_{m}\right\}, C_{i} \subseteq X,\left|C_{i}\right| \leq k$ of subsets of $X$ of size at most $k$. The objective of $k-S C$ is to find a subset $\mathcal{S} \subseteq C$ of minimum cardinality (or cost) that covers all elements of $X$, i.e., $\cup_{S_{i} \in \mathcal{S}} S_{i}=X$.

$\mathrm{k}-\mathrm{SC}$ is a well studied problem, and many approximation algorithms can be found in the literature. It is known that the greedy algorithm gives a $H_{k}$-approximation, where $H_{k}=\sum_{i=1}^{k} 1 / i$ is the $k$-th harmonic number [Garey and Johnson 2002]. Hence, this gives an easy $\frac{25}{12}$-approximation for OWN-BC, since $k=4$. More involved approximation algorithms have been developed and the current state-of-the-art for k-SC is an $\left(H_{k}-\frac{196}{390}\right)$ approximation [Levin 2008], resulting in a 1.58-approximation for OWN-BC.

During our simulations, we observed that frequently only a small fraction of the connected components can be covered by 3 - or 4 -sets. Motivated by this observation, we propose the Algorithm 1, that guarantees a factor-2 approximation in the worst case, yielding optimal solutions in certain instances.

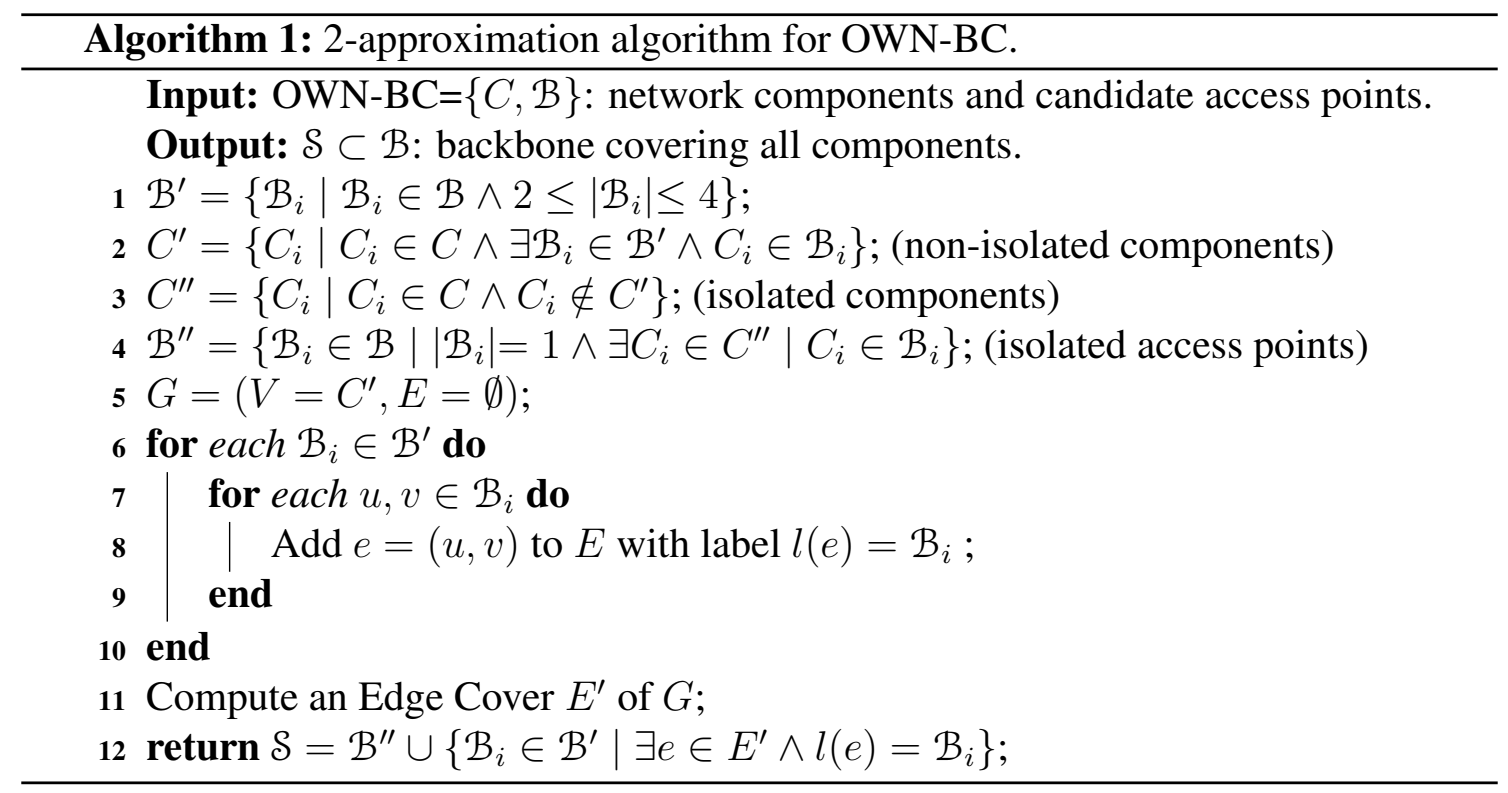

Algorithm 1 works as follows. Given an instance of the OWN-BC problem, it builds an instance of the Edge Cover problem. We show that Algorithm 1 is actually a factor-2 approximation that runs in polynomial time, which is proved in Theorem 2. 
Theorem 2. Algorithm 1 gives a 2-approximation for $O W N-B C$ in polynomial time.

Proof. Since a minimum edge cover can be found in polynomial time [Garey and Johnson 2002]), the algorithm clearly runs in polynomial time.

For the proof of the approximation factor, let $C^{\prime}$ be a solution given by Algorithm 1. Note that an optimal solution for 4-Set Cover $C^{*}$ can be converted to an edge cover $E^{*}$ of the constructed graph as follows: For each 1 -set $S$, take any edge containing $S$ as subset; For each 2-set $S$, take the edge corresponding to it; For each 3-set $S$, take two distinct edges with endpoints in $S$; For each 4-set $S$, take two disjoint edges with endpoints in $S$.

Note that $E^{*}$ is an edge cover with at most twice the number of elements of $C^{*}$. Since the edge cover $E^{\prime}$ is minimum, hence we have $\left|C^{\prime}\right| \leq\left|E^{\prime}\right| \leq\left|E^{*}\right| \leq 2\left|C^{*}\right|$, which completes the proof.

\subsection{Characterizing polynomial complexity instances}

In some cases, the OWN-BC problem reduces to the Edge Cover problem and can be solved optimally in polynomial time by Algorithm 1. We characterize OWN-BC instances in the Random OWN model and measure the probability $P_{\text {poly }}$ of the event polynomial connectivity with a backbone. We formulate a lower bound on the probability $P_{\mathrm{poly}}$.

Theorem 3. Given an instance $O W N-B C(g, \epsilon, r, \mu)$ in the Random OWN model, the probability of polynomial connectivity with a backbone can be lower bounded as follows:

$$
P_{p \circ I_{y}} \geq p(\epsilon, r, \mu)^{g^{2}}
$$

where $p(\epsilon, r, \mu)$ is defined in (5);

Proof. We need that candidate access points at all $g^{2}$ grid intersections connect at most 2 network components. We denote as $p$ the probability that this condition is satisfied at a given street intersection. Since these events at different intersections are independent, we have that $P_{\text {poly }}=p^{g^{2}}$. Using the law of total probability, the probability $p$ can be expressed as the sum over the partitions:

$$
p(\epsilon, r, \mu)=p_{+}(\epsilon, r, \mu)+2 p_{\mid}(\epsilon, r, \mu)+p_{\diamond}(\epsilon, r, \mu)
$$

We obtain a lower bound for each probability $p_{+}, p_{\mid}$and $p_{\diamond}$ [Neto et al. 2017].

\subsection{Experimental results}

We simulated several instances of the Random OWN model by using different combinations of parameters $g, \mu, \epsilon$ and $r$. The $x$-axis represents the increasing transmission range $r$ in all plots. Each value in the plot represents the mean value over 200 samples, with the respective $95 \%$ confidence interval. We did not use any simulation engines. The source code is available at goo.gl/dHpac9 and it was written in $\mathrm{C}++$ and $\mathrm{R}$ programming languages.

We show some results in the plots of Figure 3. In Figure 3a, we plot the empirical and the analytical probability $p$ (expression 5). We can see that the analytic lower-bound 


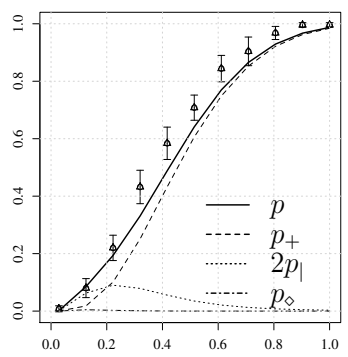

(a) Street intersection

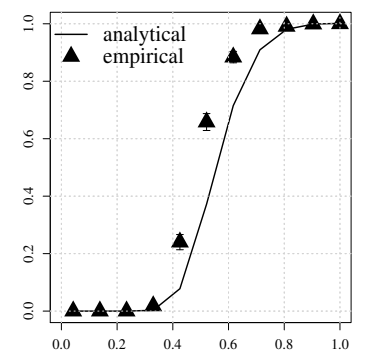

(b) Whole grid.

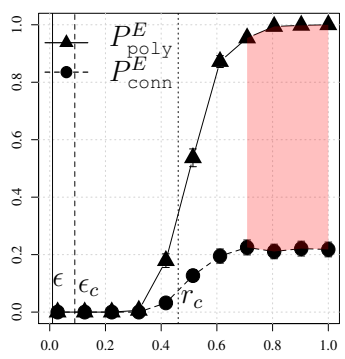

(c) $g=5, \mu=10, \epsilon=0.01$

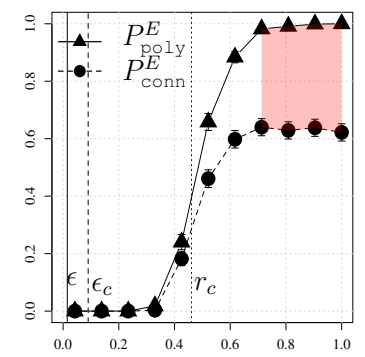

(d) $g=5, \mu=10, \epsilon=0.015$

Figure 3: Probability of polynomial connectivity with a backbone: analytical (lines) $\times$ empirical (triangles). Empirical Probability of "polynomial connectivity w/ backbone" $\left(P_{\mathrm{poly}}^{E}\right)$ and "ad hoc" $\left(P_{\mathrm{conn}}^{E}\right)$ connectivity. Shaded region: $\geq 95 \%$ optimal backbone.

is well-adjusted to the simulated data. In Figure $3 b$, we compare the empirical and the analytical lower bound for $P_{\text {poly }}$ (expression 4), which is dependent on the size of the grid $g$. In Figure $3 b$, we can see that the data adjusts well to the analytic expression 4. It should be noted that we can clearly distinguish regions of zero probability as opposed to those of probability one and that the curve captures very well these different regions.

In Figures $3 \mathrm{c}$ and $3 \mathrm{~d}$, we plot the empirical probability $P_{\mathrm{poly}}^{E}$ of the event of polynomial connectivity with a backbone, as well as $P_{\mathrm{conn}}^{E}$, the empirical probability of connectivity without a backbone infrastructure, i.e., the probability of connectivity of a ad hoc Random OWN. The area indicated between these curves represents the region in which our work shows its strength. For these regions, in addition to demonstrating that the backbone is essential, we offer an algorithm to compute close-to-optimum solutions in polynomial time to a NP-complete problem.

\section{Conclusion and Acknowledgements}

In this work we analyzed how connectivity scales in large ad hoc obstructed wireless networks and focused on the problem of establishing connectivity using a connected backbone infrastructure in sub-critical scenarios. This work was supported by CNPq, Fapemig and CAPES.

\section{References}

[Almiron et al. 2013] Almiron, M. G., Goussevskaia, O., Loureiro, A. A., and Rolim, J. (2013). Connectivity in obstructed wireless networks: From geometry to percolation. In Proc.of ACM, MobiHoc'13, pages 157-166, New York, NY, USA. ACM.

[Garey and Johnson 2002] Garey, M. R. and Johnson, D. S. (2002). Computers and intractability, volume 29. wh freeman New York.

[Levin 2008] Levin, A. (2008). Approximating the unweighted k-set cover problem: greedy meets local search. SIAM Journal on Discrete Mathematics, 23(1):251-264.

[Neto et al. 2017] Neto, M. F., Goussevskaia, O., and dos Santos, V. F. (2017). Connectivity with backbone structures in obstructed wireless networks. Computer Networks, 127:266 - 281 . 